\title{
A Modified Newcastle-Ottawa Scale for the Assessment of Study Quality in Genetic Urological Research
}

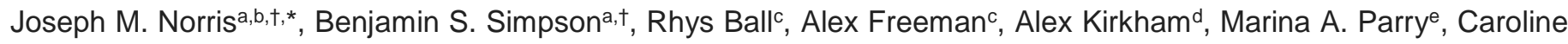
M. Moore ${ }^{a, b, \ddagger}$, Hayley C. Whitaker ${ }^{a, \ddagger}$, Mark Emberton $a, b, \ddagger$

†These authors are joint first authors.

¥ These authors are joint senior authors.

a UCL Division of Surgery \& Interventional Science, University College London, London, UK

b Department of Urology, University College London Hospitals NHS Foundation Trust, London, UK

c Department of Pathology, University College London Hospitals NHS Foundation Trust, London, UK

${ }^{\mathrm{d}}$ Department of Radiology, University College London Hospitals NHS Foundation Trust, London, UK

e UCL Cancer Institute, University College London, London, UK

* Corresponding author. UCL Division of Surgery \& Interventional Science, $3^{\text {rd }}$ Floor, Charles Bell House, 43-45 Foley

Street, London W1W 7TS, UK. Tel. +44 7724022171. E-mail address: joseph.norris@ucl.ac.uk (J.M. Norris).

Article type: Research Letter.

Word count: 481 words.

Ethics: Not required.

Funding: Joseph M. Norris is funded by the Medical Research Council (MRC) (Grant Reference: MR/S00680X/1). Benjamin S. Simpson is funded by the Rosetrees Trust (Grant Reference: M621).

Keywords: Genomics; Multiparametric MRI; Prostate cancer; Study bias.

Conflicts of interest: Norris receives funding from the MRC. Simpson receives funding from the Rosetrees Trust. Whitaker receives funding from Prostate Cancer UK, the Urology Foundation and the Rosetrees Trust. Moore is supported by the UK NIHR, Movember, PCUK and the EAU Research Foundation. Freeman, Kirkham and Emberton have stock interest in Nuada Medical Ltd. Emberton receives funding from NIHR-i4i, MRC, Sonacare Inc., Trod Medical, Cancer Vaccine Institute and Sophiris Biocorp for trials in prostate cancer. Emberton is a medical consultant to Sonacare Inc., Sophiris Biocorp, Steba Biotech, GSK, Exact Imaging and Profound Medical. Travel allowance was previously provided from Sanofi Aventis, Astellas, GSK, and Sonacare. Emberton is a proctor for HIFU with Sonacare Inc. and is paid for training other surgeons in this procedure. 
The Newcastle-Ottawa scale is a risk-of-bias tool for assessing observational studies on the basis of patient selection, comparability and outcome [1]. We recently published a review of the genetic landscape of prostate cancer conspicuity on multiparametric magnetic resonance imaging (mpMRI). In this review, we developed an adapted version of the Newcastle-Ottawa scale, in which criteria for patient selection and outcome were altered to assess methodological quality of genetic and molecular-based studies [2,3]. Here, we describe these adjustments for use in future reviews.

In the traditional Newcastle-Ottawa scale, patient selection is assessed by ascertaining representativeness of the selected cohort [1]. In our modification (Table 1), with an emphasis on genetic studies, we considered large unbiased sampling (e.g. prostate biopsy cohorts) to be most representative (encompassing the widest population cross-section), followed by smaller cohorts $(n<50)$, then tissue sampling methods with associated selection bias (e.g. radical prostatectomy cohorts), and finally, alternative non-specific sampling (e.g. transurethral resection of the prostate) was considered least representative. Whilst biopsy is potentially limited by sampling density, access route and inconsistent image-guidance, this approach still remains the most widely-accepted diagnostic modality for many cancers (especially, prostate) and as such, provides good representation of the available population, avoiding selection biases associated with more invasive techniques (e.g. prostatectomy). Whole-mount radical prostatectomy provides maximum tumour volume for interrogation, but is subject to several drawbacks that lower the degree of population representation, including, selection bias (i.e. predominantly men with organ-confined high-grade disease, who are fit enough for radical surgery), registration challenges, ex-vivo tissue shrinkage, distortion and tissue loss from trimming. For outcome assessment, we simplified the Newcastle-Ottawa scale to a single parameter, describing quality of sequencing used genetic outcomes. We considered unbiased whole genome, epigenome, transcriptome or proteome-based approaches to be highest quality (two stars), followed by limited large-scale methods based on arrays (one star), then selected gene panels (e.g. commercial assays), and finally, approaches investigating single genes were considered lowest quality. Using large-scale, next-generation sequencing may appear contradictory to hypothesis-driven research (which favours candidate molecules), however, investigator-hypotheses based on single candidate genes are actually subject to additional biases. Firstly, publication bias occurs, whereby non-significant findings are supressed, and significant findings are reported; as such, molecular correlates with 'significant' results become targets for further research, resulting in confirmation bias. Furthermore, richly annotated genes with clearly elucidated functions are naturally subject to further investigation, a phenomenon that has increased over time, leading to further publications and a compounding effect [4]. In fact, only approximately $10 \%$ of all known human genes are commonly included in research, and this may simply be due to favourable research characteristics, allowing ease of study [5]. For this reason, we have cited largescale techniques such as RNAseq as having lower risk of bias than microarray techniques, as they allow hypothesis testing without prohibiting investigation of alternative possibilities. This also enables exploration of non-coding transcripts and transcript variants, as opposed to only protein-coding genes. 
We hope our modified Newcastle-Ottawa scale (Table 1) will provide future researchers with a valuable tool when reviewing genetic-based studies in urology. 
Table 1 - A modified Newcastle-Ottawa scale for genetic studies. Good quality: 3/4 stars for Selection, $1 / 2$ stars for Comparability, 2/3 stars for Outcome. Fair quality: 2 stars for Selection, 1/2 stars for Comparability, 2/3 stars for Outcome. Poor quality: 0/1 star for Selection, 0 stars for Comparability, 1 star for Outcome.

\begin{tabular}{|c|c|}
\hline Category & Scoring criteria \\
\hline \multirow[t]{4}{*}{$\begin{array}{l}\text { Selection } \\
\text { (max. } 4 \text { stars) }\end{array}$} & $\begin{array}{l}\text { Representativeness of exposed group (max. } 1 \text { star) } \\
\text { - Wide-spectrum tissue sampling; } \\
\text { low selection bias (e.g. prostate biopsy) (1 star) } \\
\text { - Large volume tissue sampling; } \\
\text { acknowledged selection bias (e.g. prostatectomy) (1 star) } \\
\text { - Highly selected group (0 stars) } \\
\text { - No description (0 stars) }\end{array}$ \\
\hline & $\begin{array}{l}\text { Selection of non-exposed group (max. } 1 \text { star) } \\
\text { - Same source population as exposed group (1 star) } \\
\text { - Drawn from different source (0 stars) } \\
\text { - No description ( } 0 \text { stars) }\end{array}$ \\
\hline & $\begin{array}{l}\text { Ascertainment of exposure (max. } 1 \text { star) } \\
\text { - Secure record (e.g. pathological record) (1 star) } \\
\text { - Structured interview ( } 1 \text { star) } \\
\text { - Written self-report (0 stars) } \\
\text { - No description (0 stars) }\end{array}$ \\
\hline & $\begin{array}{l}\text { Outcome demonstrably absent at baseline (max. } 1 \text { star) } \\
\text { - Yes (1 star) } \\
\text { - No (0 stars) }\end{array}$ \\
\hline $\begin{array}{l}\text { Comparability } \\
\text { (max. } 2 \text { stars) }\end{array}$ & $\begin{array}{l}\text { Comparability of cohorts, controlling for confounders } \\
\text { - Controls for key confounders (e.g. Gleason grade) ( } 1 \text { star) } \\
\text { - Controls for related factors (e.g. tumour size) ( } 1 \text { star) } \\
\text { - Cohorts incomparable, confounders uncontrolled (0 stars) }\end{array}$ \\
\hline $\begin{array}{l}\text { Outcome } \\
\text { (max. } 2 \text { stars) }\end{array}$ & $\begin{array}{l}\text { Outcome assessment } \\
\text { - Genome/epigenome/transcriptome/proteome ( } 2 \text { stars) } \\
\text { - Large arrays/panels (> } 100 \text { genes) ( } 1 \text { star) } \\
\text { - Targeted panels (< } 100 \text { genes) ( } 0 \text { stars) } \\
\text { - Small panel ( } 0 \text { stars) } \\
\text { - Single target ( } 0 \text { stars) }\end{array}$ \\
\hline
\end{tabular}

Max = maximum score. 


\section{References}

[1] Wells GA, Shea B, O'Connell D, et al. The Newcastle-Ottawa Scale (NOS) for assessing the quality if nonrandomized studies in meta-analyses. Available from: ohri.ca/programs/clinical_epidemiology/oxford.htm

[2] Norris JM, Simpson BS, Parry MA, et al. Genetic landscape of prostate cancer conspicuity on multiparametric MRI: a protocol for a systematic review and bioinformatic analysis. BMJ Open 2020;10:e034611.

[3] Norris JM, Simpson BS, Parry MA, et al. Genetic landscape of prostate cancer conspicuity on multiparametric magnetic resonance imaging: a systematic review and bioinformatic analysis. Eur Urol Open Sci 2020;20:3747.

[4] Haynes WA, Tomczak A, Khatri P. Gene annotation bias impedes biomedical research. Sci Rep 2018;8:1362.

[5] Stoeger T, Gerlach M, Morimoto RI, et al. Large-scale investigation of the reasons why potentially important genes are ignored. PLoS Biol 2018;16:e2006643. 